\title{
Thermodynamics of a Non-Stationary Black Hole Based on Generalized Uncertainty Principle
}

\author{
Mustari and Yuant Tiandho* \\ Department of Physics, Universitas Bangka Belitung \\ Jl. Kampus Peradaban, Merawang, Kep. Bangka Belitung \\ E-mail: yuant@ubb.ac.id
}

Received 29 August 2017, Revised 27 September 2017, Accepted 2 October 2017

\begin{abstract}
In the general theory of relativity (GTR), black holes are defined as objects with very strong gravitational fields even light can not escape. Therefore, according to GTR black hole can be viewed as a nonthermodynamic object. The worldview of a black hole began to change since Hawking involves quantum field theory to study black holes and found that black holes have temperatures that analogous to black body radiation. In the theory of quantum gravity there is a term of the minimum length of an object known as the Planck length that demands a revision of Heisenberg's uncertainty principle into a Generalized Uncertainty Principle (GUP). Based on the relationship between the momentum uncertainty and the characteristic energy of the photons emitted by a black hole, the temperature and entropy of the non-stationary black hole (Vaidya-Bonner black hole) were calculated. The non-stationary black hole was chosen because it more realistic than static black holes to describe radiation phenomena. Because the black hole is dynamic then thermodynamics studies are conducted on both black hole horizons: the apparent horizon and its event horizon. The results showed that the dominant correction term of the temperature and entropy of the Vaidya-Bonner black hole are logarithmic.
\end{abstract}

Keyword: Black hole, thermodynamics, generalized uncertainty principle.

\section{INTRODUCTION}

One of the exotic phenomena that directly formulated through the general theory of relativity (GTR) is a black hole. In GTR, black hole can be defined as a region of spacetime with very strong gravity so that incoming light will not escape from it (Frolov \& Novikov, 1998). So in a classical theory, it is safe to say a black holes can not radiate or emite any particles (Tiandho, 2017). However, by using the quantum mechanics, Hawking has proved that black holes are not so black. This is because a black hole have different properties, unlike those depicted in GTR. Through his research, Hawking has shown that the black hole can radiate particles like a black body radiation with temperature (in a natural unit) as (Hawking, 1975),

$$
T=\frac{1}{8 \pi M}
$$

In a recently decades the study of black hole thermodynamics has been a hot topic in quantum gravity. One type of black hole that is interesting to discuss is the Vaidya- 
Bonner black hole. The Vaidya-Bonner black hole is a non-stationary black hole. The non-stationary of the Vaidya-Bonner black hole is represented by its mass and charge that depend on space and time. So the Vaidya-Bonner black hole is expected to provide a more realistic picture of the black hole radiation process. Some methods that can be used to calculate the temperature of thermodynamic black holes are the Damour-Ruffini method (Liu, 2011), Teukolsky's method (Kim, Choi, Kim, \& \& Yang), second quantization method (Yang, 1995), radial method null geodesic (Parikh, 2000), complex path method (Srinivasan \& Padmanabhan, 1989), and according to relation between Heisenberg's uncertainty principle with the energy-momentum dispersed particle. The latter method is a relatively simple method compared to the others. However, since the black hole thermodynamic study is one of the topics studied in quantum gravity theory it should be calculating the temperature of black holes using the basic principles in the theory. This is because in theory quantum gravity is known the minimum length limit of an object called the Planck length. So in the theory of quantum gravity the principle of uncertainty must be modified into a generalized uncertainty principle (GUP). The muchstudied GUP form contains the momentum as the quadratic term (L.J. Garay, 1995),

$$
\delta x \delta p \geq \hbar \quad \begin{array}{cc}
-.21 & -2
\end{array}
$$

with $\delta x$ and $\delta p$ each of which is the uncertainty of the position and momentum of the quantum particles, $l_{p}$ is the length of Planck and $\alpha$ are constants that are not dimensionless and depend on the model that used.

Therefore, for the study of black hole temperatures can accommodate the Planck lenght correction factor in quantum gravity theory, a study of black hole temperatures that previously used the Heisenberg uncertainty principle should be corrected. In this paper will be calculated thermodynamic black hole by using GUP correction. The black hole studied is the Vaidya-Bonner black hole with its radius should be much larger than the length of Planck. This approach is done because most black holes found in the universe have a very large size, for example the holes found in the center of the galaxy have a radius of about $1,25 \times 10^{10} \mathrm{~m}$.

\section{Vaidya-Bonner Black Hole}

The Vaidya-Bonner black hole is a non-stationary black hole in which its mass and charge are generally a function of advanced time (u) (Ibohal \& Kapil, 2010),

$$
M=M(u) ; \quad Q=Q(u)
$$

The solution of the space-time metrics of Vaidya-Bonner is,

$$
d s^{2}=-f d u^{2}+2 d u d t+r^{2} d \Omega^{2}
$$

where $f=1-\frac{2 M(u)}{r}+\frac{Q(u)}{r^{2}}$ and $d \Omega^{2}=d \theta^{2}+\sin ^{2} \theta d \varphi^{2}$. When mass and charge are not functions $u$ or a constant value, the above metrics will reduce to the ReissnerNordstrom metric. As for if $Q=0$ it is clear that the metric will reduce to Vaidya's 
metrics for $M=M(u)$ or would be a Schwarzschild metric if its mass is constant. Unlike in static black holes, event horizon and apparent horizon in a non-stationary black holes lies in different places. For Vaidya-Bonner's black hole, its apparent horizon lies on (Niu \& Liu, 2010)

$$
r_{A H}=M+\sqrt{M^{2}-Q^{2}}
$$

While the event horizon lies on (Zheng \& Xianxin, 1992),

$$
r_{E H}=\frac{M+\sqrt{M^{2}-(1-2 i}}{1-2 i}
$$

where $i_{\text {un }}$. It can concluded for conditions $i^{\circ}$ the location of event horizon and apparent horizon will be have same position and this is the fingerprint of a static black hole. Thus, since the event horizon and apparent horizon are located in different places, this paper we will study the thermodynamics of both horizons.

\section{Formulation of Black Hole Entropy Based on Generalized Uncertainty Principle}

By slightly manipulating the GUP equation, then we can specify the variables in the GUP equation as follows (with $\hbar$ ),

$$
\delta p \geq \frac{\delta x}{2 \alpha^{2} l_{p}^{2}}\left[1-\sqrt{1-\frac{4 \alpha^{2} l_{p}^{2}}{\delta x^{2}}}\right]
$$

The minus sign in the above equation is selected for $l_{p}^{2} \rightarrow 0$ then the solution obtained will reduce to the classical uncertainty principle (Heisenberg's principle). Because in this paper black hole to be analyzed has a very large mass, then the correction terms $l_{p}^{2} / 2$ it so small. So that the momentum in uncertainty formula can be expanded according to the Taylor series,

$$
\delta p \geq \frac{1}{\delta x}\left[1+\left(\frac{\alpha^{2} l_{p}^{2}}{\delta x^{2}}\right)+2\left(\frac{\alpha^{2} l_{p}^{2}}{\delta x^{2}}\right)^{2}+O\left(l_{p}\right)^{5}+\ldots\right]
$$

In previous research, it has been shown that momentum uncertainty is related to the particle characteristic energy that emitted from black holes. Some calculations in quantum gravity theory also support the study of the energy-moment dispersion relationship for high energy particles which can be expressed as (Camelia, Arzano, \& Procaccini, 2004; Gambini. \& Pullin, 1999)

$$
E \simeq \quad+\beta l_{p} E^{2}
$$


where $\beta$ is a coefficient of order 1 . Since the particles emitted from the black hole are much smaller than the mass of the black hole (in some calculations also using massless particles), the particle emissions energy and momentum have a relationship $E \approx p$. Thus expressing the energy of emitted particles can be expressed as,

$$
E \geq \frac{1}{\delta x}\left[1+\left(\frac{\alpha^{2} l_{p}^{2}}{\delta x^{2}}\right)+2\left(\frac{\alpha^{2} l_{p}^{2}}{\delta x^{2}}\right)^{2}+O\left(l_{p}\right)^{5}+\ldots\right]
$$

This result is consistent with the results obtained in the quantum gravity loop theory and the particle dispersion energy can be expressed as $E \geq\left(1+2 \beta l_{p} / \delta x\right) / \delta x$ (Camelia, Arzano, \& Procaccini, 2004). It appears that in a standard dispersion relationships (without Planck scale correction), eq. (10) will be reduced to the Heisenberg uncertainty principle (in natural unit). The standard dispersion relationship was then used by Bekenstein to calculate the entropy of black holes. When a black hole emits particles and absorbs particles whose energy $E$ and size $R$, then the area change can be expressed as (Medved \& Vagenas, 2004)

$$
(\Delta A)_{\min } \geq 8 \pi l_{p}^{2} E R
$$

In classical studies we may use $R=0$ but when involved in minimum lengths then quantum particles will never be smaller than $\delta x$, which is the intrinsic uncertainty of the particle's position. Therefore, through the GUP we can write the change of the black hole area as,

Through substitution, eq. (12) into eq. (10) will be obtained,

$$
\Delta A_{\min } \geq 8 \pi l_{p}^{2}\left[1+\frac{\alpha^{2} l_{p}^{2}}{\delta x^{2}}+2\left(\frac{\alpha^{2} l_{p}^{2}}{\delta x^{2}}\right)^{2}+O\left(l_{p}\right)^{5}+\ldots\right]
$$

In Hawking's temperature calculations by using the uncertainty principle, coefficient $\delta x$ in Schwarzschild's black hole is related to the event horizon, $\delta x=\gamma \pi r_{h}$, and $\gamma$ is a calibration factor and can be determined from the relationship between Hawking temperature which obtained from the uncertainty principle and the conventional Hawking temperature, that is $\gamma=2 \pi$ (Alder, Chen, \& Santiago, 2001) So, according to the definition of black hole area eq. (9) can be expressed in form, 


$$
\Delta A_{\min } \geq 8 \pi l_{p}^{2}\left[1+\frac{\alpha^{2} l_{p}^{2}}{\pi A}+2\left(\frac{\alpha^{2} l_{p}^{2}}{\pi A}\right)^{2}+O\left(l_{p}\right)^{5}+\ldots\right]
$$

Basically the entropy of a black hole is a measure of information about the interior of a black hole that is not accessible to an exterior observer (Bekenstein, 1973). So based on the notion of information theory, it may be argued that the increment of minimal entropy is independent on the area or it can be called as a "bit" information, and the entropy of the fundamental unit can be expressed as $b$. If the relationship between the change of area and entropy is,

$$
\frac{d S}{d A}=\frac{\Delta S_{\min }}{\Delta A_{\min }}=\frac{b}{8 \pi l_{p}^{2}\left(1+\frac{\alpha^{2} l_{p}^{2}}{A \pi}+2\left(\frac{\alpha^{2} l_{p}^{2}}{A \pi}\right)^{2}+O\left(l_{p}\right)^{5}+\ldots\right)}
$$

Thus, according to the Taylor series the above expression can be expanded as,

$$
\left.\frac{d S}{d A} \simeq \operatorname{unp}_{\ln }-\left(\frac{\alpha^{2} l_{p}^{2}}{A \pi}\right)-\left(\frac{\alpha^{2} l_{p}^{2}}{A \pi}\right)^{2}+3\left(\frac{\alpha^{2} l_{p}^{2}}{A \pi}\right)^{3}+O\left(l_{p}\right)^{5}+\ldots\right)
$$

If the entropy is in eq. (12) integration will be obtained,

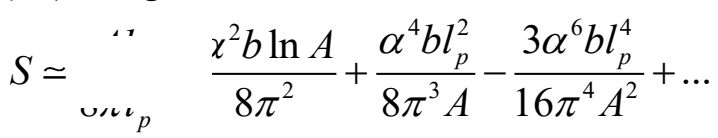

Therefore if the corrected entropy is defined as, $S=S_{0}+S_{c}$, with $S_{0}=A / 4 l_{p}^{2}$ is Bekenstein entropy (in natural unit) and $S c$ is a entropy correction term hence can be determined $b=2 \pi$, so the result will reduce to Bekenstein entropy when it not involved Planck scale correction factor. Thus, the corrected entropy in explicit form is

$$
S=\frac{A}{4 l_{p}^{2}}-\frac{\alpha^{2} \ln A}{4 \pi}+\frac{A}{4 l_{p}^{2}}\left[\sum_{i=1} c_{i}\left(\frac{\alpha^{2} l_{p}^{2}}{\pi A}\right)^{i+1}\right]
$$

\section{Thermodynamics on Apparent Horizon}

By considering the definition of apparent horizon as in eq. (5) then entropy on eq. (18) can explicitly be expressed as, 


$$
\begin{gathered}
\left.S_{A H}=\frac{\pi\left(M+\sqrt{M^{2}-Q^{2}}\right)}{l_{p}^{2}}-\frac{\ln \left(4 \pi\left(M+\sqrt{M^{2}-Q^{2}}\right)^{2}\right)}{4 \pi}\right)^{+\frac{\pi\left(M+\sqrt{M^{2}-Q^{2}}\right)}{l_{p}^{2}}}\left[\sum_{i=1} c_{i}\left(\frac{\alpha^{2} l_{p}^{2}}{\pi\left(\frac{\left.\pi+\sqrt{M^{2}-Q^{2}}\right)}{l_{p}^{2}}\right)}\right)^{i+1}\right]
\end{gathered}
$$

It appears that explicitly entropy on apparent horizon when involving GUP correction contains logarithmic correction terms (for the most dominant terms). The graph of the relationship between the black hole entropy with its mass and its charge can be seen in Figure 1. Through Figure 1 it can be seen that the greater black hole mass so the higher its entropy. As for the charge (at a constant mass) the greater black hole charge so the lower its entropy. Another interesting point is that there is an entropy condition that is not allowed in a black hole when the charge of a black hole is larger than its mass. This is related to extremal black hole conditions $(M=Q)$. In extremal conditions, the entropy of the black hole on the apparent horizon will reduce in form (three terms correction),

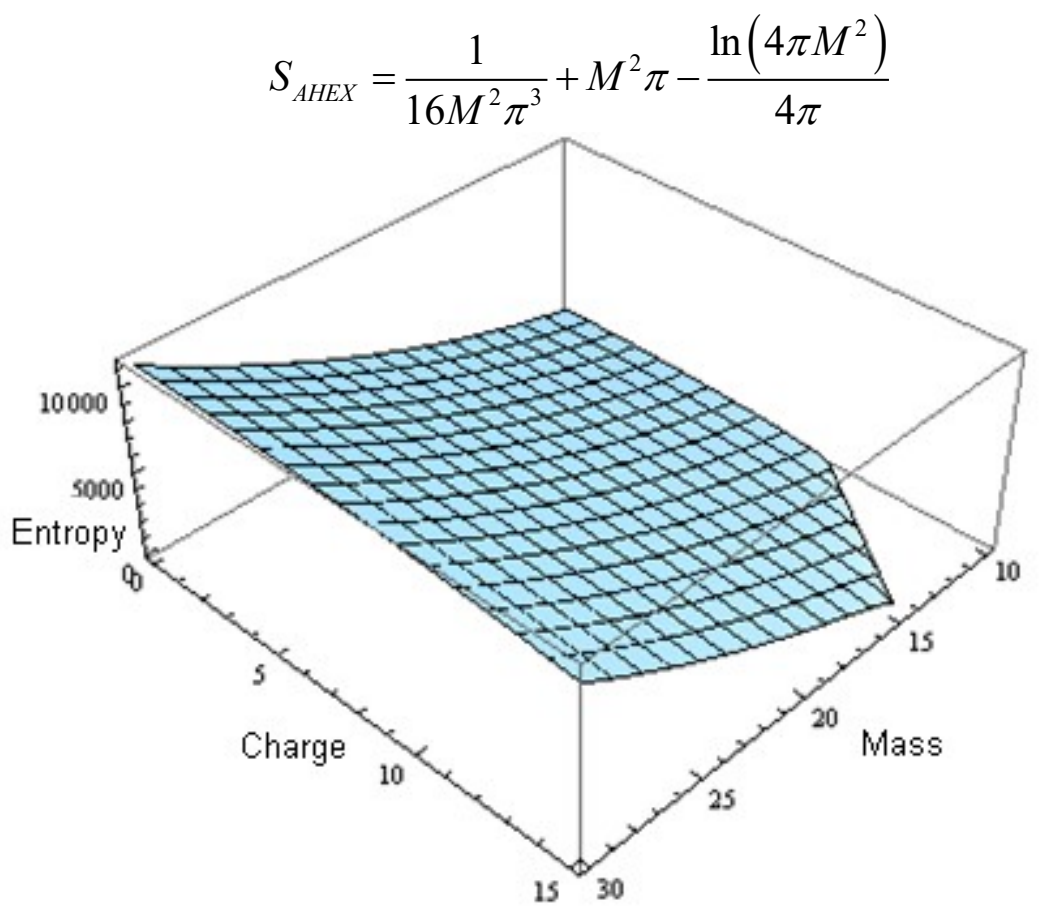

Figure 1. Graph of relationship between entropy with mass and charge of black hole on apparent horizon based on GUP (for three correction terms and $l_{p}=\alpha=1$ )

The temperature on the apparent horizon in the Vaidya-Bonner black hole can be determined by the first law of black hole thermodynamics (assuming emission particles are uncharged) 


$$
\frac{1}{T}=\frac{\partial S}{\partial M}
$$

So with the definition of entropy as in eq. (19) then the temperature of the VaidyaBonner black hole on the apparent horizon can be expressed as (three correction terms),

$$
T_{A H}=\frac{8 \pi^{3}\left(M+\sqrt{M^{2}-Q^{2}}\right)^{3}}{\left(1+\frac{M}{\sqrt{M^{2}-Q^{2}}}\right)\left(-1-4 \pi^{2}\left(M+\sqrt{M^{2}-Q^{2}}\right)^{2}+16 \pi^{4}\left(M+\sqrt{M^{2}-Q^{2}}\right)^{4}\right)}
$$

The graph of the relationship between black hole temperature and its mass and its charge is given by Figure 2. In Figure 2 it appears that the higher black hole mass the black hole's temperature on the apparent horizon will be smaller as well as the charge. When be carefully considered, there is a condition of the temperature drop to zero that occurs at the value $M=Q$. This can happen because the condition is an extremal black hole condition. So through this result it can be concluded that the temperature calculations using GUP corrections on apparent horizon still satisfy the third law of black hole thermodynamics.

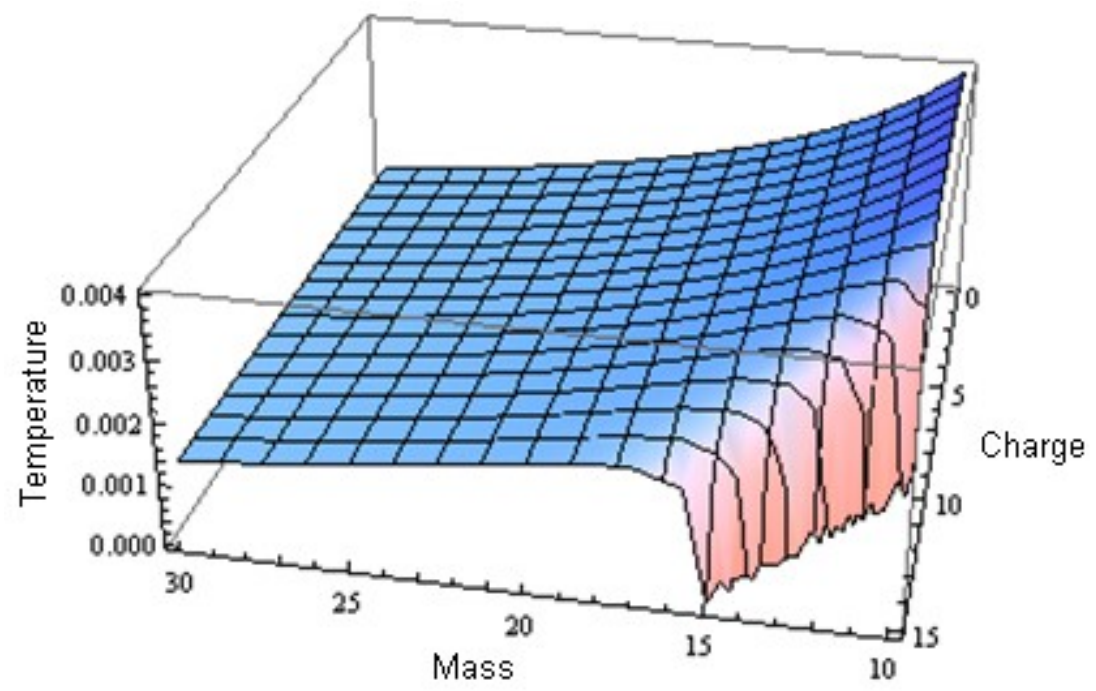

Figure 2. The relationship between black hole temperature on the apparent horizon with its mass and its charge based on the GUP correction (for three correction terms and $l_{p}=$ $\alpha=1)$.

\section{Thermodynamics on Event Horizon}

Through the formulation of event horizon on eq. (6) then the entropy on the horizon event can be expressed explicitly as (three corrected terms), 


$$
\begin{aligned}
S_{E H}= & \alpha^{2} \ln \left(\frac{4 \pi\left[M+\sqrt{M^{2}+Q^{2}(-1+2 i}\right.}{4 \pi} \quad\right)^{\prime} \frac{(1-2 i}{16 \pi^{2}\left(M+\sqrt{M^{2}+Q^{2}(1-2 i}\right.} \overline{-} \\
& +\frac{\pi\left(1 6 \pi ^ { 2 } \left(M+\sqrt{M^{2}+Q^{2}(1-2 i}\right.\right.}{(1-2 i} .
\end{aligned}
$$

As with the entropy on the apparent horizon it can be seen that the most dominant correction term in the entropy on the event horizon is related to logarithmic functions. It can be seen that the entropy of black hole at the event horizon depends on its mass and its charge also depends on the speed of change of the event horizon. So that according to results, we need condition in order to the entropy is not to go infinite because basically the denominator contains the speed of event horizon change variable which should not be $1 / 2$. To be able to do interpretation of the value then we require further study related to the process of changing event horizon radius, especially that involves aspects of the speed of change.

As for the temperature of Vaidya-Bonner black hole on the event horizon can also be determined based on the first law of thermodynamics as in eq. (21) and it can be read as (three tems correction),

$T_{E H}=\left(8 \pi^{2}\left(M+\sqrt{M^{2}+Q^{2}(-1+2}\right.\right.$.

$$
/\left(1+\frac{M}{\sqrt{M^{2}+Q^{2}(-1+2 i}}\right)_{-}\left(1-2 l_{p i}\right.
$$$$
/ \quad+\frac{16 \pi^{3}\left(M+\sqrt{M^{2}+Q^{2}(-1+2 i}\right.}{\left(1-2 l_{p} r_{e h}\right)^{2}}
$$

From the above equation it appears that the temperature of the black hole in the event horizon depends on the mass, the charge, and the speed of the event horizon change. If the speed of the event horizon change is zero then the above equation will reduce to temperature at the apparent horizon. As with the apparent horizon, in extremal conditions the temperature at the horizon will lead to zero to satisfy the third law of thermodynamic conditions. If we look at the formulation of entropy and temperature at the event horizon then we need further study either geometry or study of mechanism of tunneling particle emission black hole to be able to answer the special value of the speed of event horizon change.

\section{Conclusions}

Based on the study that has been done can be concluded that the correction of the uncertainty principle into the GUP to calculate the entropy of black hole will modify the formulation of the conventional black hole entropy. The GUP correction results show that the dominant term on the entropy is a logarithmic functions. On the apparent horizon the incresing of black hole mass will be followed by the entropy. As for the 
charge, the greater its charge hence the entropy on the apparent horizon gets smaller. The temperature on the apparent horizon is inversely proportional to the mass and charge of the black hole. Thermodynamics conditions on the event horizon depends on the speed of event horizon changes in the advanced time. There is a logarithmic correction term on the entropy on the event horizon such as the apparent horizon. There is a certain value in the speed of event horizon change which causes the entropy value and temperature at the event horizon to be unreal. So a follow-up study of these results is required. However, based on the assessment of the temperature on the apparent horizon and the event horizon it can be concluded that both results still satisfy the third law of thermodynamics black hole.

\section{Suggestions}

Through the results, the suggestion for further research is to conduct a study related to the change of event horizon and to conduct study related to the effect of entropy and temperature correction on the characteristics of the remaining black hole.

\section{Acknowledgements}

This research was funded by Universitas Bangka Belitung.

\section{References}

Alder, R., Chen, P., \& Santiago, I. (2001). The generalized uncertainty principle and black hole remnants. General Relativity and Gravitation , 33, no 12, 21012108.

Bekenstein, J. D. (1973). Black holes and entropy. Physical Review D , 7, no 8, 23332346.

Camelia, A., Arzano, M., \& Procaccini, A. (2004). Severe constraints on the loopquantum-gravity energy-momentum dispersion relation from the black-hole area-entropy law,. Physical Review , 70, 107201.

Frolov, V. P., \& Novikov, I. D. (1998). Black Hole Physics: Basic Concepts And New Developments. Netherland: Kluwer Publishing.

Gambini., R., \& Pullin, J. (1999). Nonstandar optics from quantum space time. Physical Review. D , 59, 124021.

Hawking, S. W. (1975). Particle Creation by Black Hole . Commun. Math Phys , 43, 199-220.

Ibohal, N., \& Kapil, L. (2010). Charged black holes in Vaidya backgrounds: Hawking radiation. Int. J. Mod. Phys. , 19 (4), 437-464.

Kim, S. W., Choi, E. Y., Kim, S., \& \& Yang, J. Black hole radiation in the Vaidya metric, . Phys, Lett. A 141, 238-241.

L.J. Garay. (1995). Quantum gravity and minimum length. International Journal of Modern Physics , 10, no 2, 145-165.

Liu, X. \&. (2011). Apparent horizon and event horizon thermodynamics of a Vaidya 
Black hole using Damour -Ruffini method. Astrophys. space., 331, 237-241.

Medved, A. J., \& Vagenas, E. C. (2004). When conceptual worlds collide: the generalized uncertainty prinsiple and the Bekenstein-hawking entropy. Physical Review , 70, 124021.

Niu, Z. F., \& Liu, W. B. (2010). Hawking radiation and thermodynamics of a VaidyaBonner black hole. Research in Astronomy and Astrophysics , 10, 33-38.

Parikh, M. K. \& Wilczek, F. (2000). Hawking radiation as tunneling. Phys. Rev. Lett. 85,5042 .

Srinivasan, K., \& Padmanabhan, T. (1999). Particle production and complex path analysis. Phys. Rev. D $60,024007$.

Tiandho, Y. (2017). Mesin Panas Foto-Carnot Lubang Hitam Non-Stasioner. Jurnal Sains Dasar , 1, 17-25.

Yang, Z. (1995). study of hawking effect in Vaidya-Bonner Black hole by means of second quantization. Acta Physica Sinica, 141, 241-247.

Zheng, Z., \& Xianxin, D. (1992). A new method with Hawking effects of evaporating black holes. Modern Physics Letters A , 7 (20), 1771-1778. 